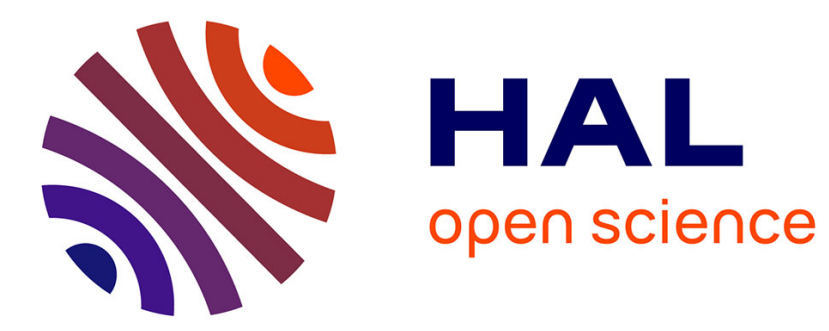

\title{
Global adaptive synchronization based upon polytopic observers
}

\author{
Floriane Anstett, Gilles Millérioux, Gérard Bloch
}

\section{To cite this version:}

Floriane Anstett, Gilles Millérioux, Gérard Bloch. Global adaptive synchronization based upon polytopic observers. May 2004, pp.728-731 (CDROM). hal-00120386

\section{HAL Id: hal-00120386 https://hal.science/hal-00120386}

Submitted on 14 Dec 2006

HAL is a multi-disciplinary open access archive for the deposit and dissemination of scientific research documents, whether they are published or not. The documents may come from teaching and research institutions in France or abroad, or from public or private research centers.
L'archive ouverte pluridisciplinaire HAL, est destinée au dépôt et à la diffusion de documents scientifiques de niveau recherche, publiés ou non, émanant des établissements d'enseignement et de recherche français ou étrangers, des laboratoires publics ou privés. 


\title{
GLOBAL ADAPTIVE SYNCHRONIZATION BASED UPON POLYTOPIC OBSERVERS
}

\author{
F. Anstett, G. Millerioux, G. Bloch \\ Centre de Recherche en Automatique de Nancy, CRAN (France) \\ Ecole Superieure des Sciences et Techniques de l'Ingenieur de Nancy (ESSTIN) \\ corresponding author:millerioux@esstin.uhp-nancy.fr
}

\begin{abstract}
A new adaptive synchronization scheme achieving a joint state and parameter estimation is proposed as an alternative to the well-known Extended Kalman Filter based on a first order approximation. It involves a polytopic observer which does no longer require a linearization. The resulting adaptive synchronization is global and holds for a large class of chaotic systems. The computation of the gain of the observer is performed in a systematic way and is derived from the solutions of a Linear Matrix Inequalities set.
\end{abstract}

\section{INTRODUCTION}

Synchronization of two systems which neither assumes identical initial conditions nor identical parameters is referred to as adaptive synchronization. Parameter mismatch is a realistic and problematic situation in engineering area while in some communication scheme the mismatch is intentional. Indeed, it is typically the case when a parameter is modulated according to a rule which encodes the information to be transmitted as in chaos shift keying.

When full state is available, an adaptive controller can be designed. In [1], an adaptive approach is proposed in order to track a slowly time-varying parameter of a chaotic system. On the other hand, when only partial state is available from the output of the chaotic system, some adaptive observer-based approaches can be used. The reader can refer to [2][3][4] in the context of chaos synchronization or [5] for a more general automatic control point of view. Besides, many works dealing with synchronization with unknown parameters involve the use of an Extended Kalman Filter (EKF) which achieves a joint state and parameter estimation to handle the problem. See for example [6] for the continuous case or [7] for the discrete case.

Nevertheless, achieving global stability of the adaptive algorithms is a challenging task. For adaptive controllers or observers, the difficulty stems from the fact that the chaotic system must verify some special properties such as nonlinearities with Lipschitz conditions, special structure such as output injection. In this paper, a new joint state and unknown parameters estimation algorithm is proposed as an alternative to the Extended Kalman Filter. The advantage lies in the fact that the observer does no longer requires a first order approximation as it does for the EKF. The convergence analysis is based on the theoretical results stated in [8]. It is shown that the resulting adaptive synchronization is global and holds for a large class of chaotic systems admitting a polytopic description. The computation of the gain of the observer is systematic and is derived from the solutions of a Linear Matrix Inequalities set.

\section{POLYTOPIC DESCRIPTION}

As a preliminary, this section aims at stating some conditions under which the dynamical matrix $\mathcal{A}$ of the general system described by

$$
\begin{aligned}
& \bar{x}_{k+1}=\mathcal{A}\left(y_{k}\right) \bar{x}_{k}+E\left(y_{k}\right) \\
& y_{k}=\bar{C} \bar{x}_{k}
\end{aligned}
$$

can be rewritten in a polytopic form. $\bar{x}_{k} \in \Re^{q}, \mathcal{A} \in \Re^{q \times q}$, $\bar{C} \in \Re^{m \times q}$. $E$ is a (possible) nonlinear $q$-dimensional function depending on $y_{k}$.

Proposition 1 If there exists a function $h: \Re^{m} \rightarrow \Re^{L}$, defined as $\rho_{k}=h\left(y_{k}\right)$ with $\rho_{k}$ being bounded when $y_{k}$ is bounded, such that $\mathcal{A}\left(y_{k}\right)=\mathcal{A}\left(\rho_{k}\right)$ holds and rendering $\mathcal{A}$ to be of class $C^{1}$ with respect to $\rho_{k}$, then there always exist some $\xi_{k}^{i}$ 's and $\bar{A}_{i}$ 's such that :

$$
\mathcal{A}\left(\rho_{k}\right)=\sum_{i=1}^{N} \xi_{k}^{i}\left(\rho_{k}\right) \bar{A}_{i}
$$

with $\bar{A}_{i}$ 's corresponding to the vertices of the convex hull $\operatorname{Co}\left\{\bar{A}_{1}, \ldots, \bar{A}_{N}\right\}$, and $\xi_{k}^{i}$ 's belonging to the compact set $\mathcal{S}=\left\{\mu_{k} \in \Re^{N}, \mu_{k}=\left(\mu_{k}^{1}, \ldots, \mu_{k}^{N}\right)^{T}, \mu_{k}^{i} \geq 0 \forall i \quad\right.$ and $\left.\sum_{i=1}^{N} \mu_{k}^{i}=1\right\}$. $\mathcal{A}$ is said to be expressed in a polytopic form and $\rho_{k}$ acts as a time-varying parameter for $\mathcal{A}$. From the definition of the output $y_{k}, \rho_{k}$ is clearly on-line available.

Proof

The proof is constructive in the sense that it explicitly provides the $\xi_{k}^{i}$ 's and the $\bar{A}_{i}$ 's. In the proof, the dependence on 
$\rho_{k}$ of the $\xi_{k}^{i}$,s will be omitted for clarity sake.

Determination of $\xi_{k}^{i}$

On one hand, since $\mathcal{A}$ is of class $C^{1}$ with respect to the entries of $\rho_{k}, \mathcal{A}$ can be rewritten in the form

$$
\mathcal{A}\left(\rho_{k}\right)=A_{0}+\sum_{j=1}^{L} \rho_{k}^{(j)} A_{j}^{l c}
$$

$A_{0}$ is the matrix derived from $\mathcal{A}\left(\rho_{k}\right)$ by keeping its constant entries while setting to zero its time-varying entries. Let $l$ and $c$ two indices depending on $j$ and corresponding respectively to the position of $\rho_{k}^{j}$ in $\mathcal{A}\left(\rho_{k}\right)$. Then, $A_{j}^{l c}$ is the matrix whose entries are all zero except the one, located at row $l$ and column $c$, which equals unity.

On the other hand, since $\rho_{k}$ is bounded, it is constrained to a compact domain which can always be embedded in a polytope and thus verifies :

$$
\rho_{k}=\sum_{i=1}^{N} \xi_{k}^{i} \bar{\rho}_{i} \quad \text { with } \quad \sum_{i=1}^{N} \xi_{k}^{i}=1, \quad \xi_{k}^{i} \geq 0
$$

$N$ corresponds to the number of vertices $\bar{\rho}_{i}$ of the polytope. Equation (4) can be rewritten in a matrix way

$$
\underbrace{\left[\begin{array}{l}
\rho_{k}^{(1)} \\
\vdots \\
\rho_{k}^{(j)} \\
\vdots \\
\rho_{k}^{(L)} \\
1
\end{array}\right]}_{E_{k}}=\underbrace{\left[\begin{array}{lllll}
\bar{\rho}_{1}^{(1)} & \ldots & \bar{\rho}_{i}^{(1)} & \ldots & \bar{\rho}_{N}^{(1)} \\
\vdots & \vdots & \vdots & \vdots & \vdots \\
\bar{\rho}_{1}^{(j)} & \ldots & \bar{\rho}_{i}^{(j)} & \ldots & \bar{\rho}_{N}^{(j)} \\
\vdots & \vdots & \vdots & \vdots & \vdots \\
\bar{\rho}_{1}^{(L)} & \ldots & \bar{\rho}_{i}^{(L)} & \ldots & \bar{\rho}_{N}^{(L)} \\
1 & \ldots & 1 & \ldots & 1
\end{array}\right]}_{F} \underbrace{\left[\begin{array}{c}
\xi_{k}^{1} \\
\ldots \\
\xi_{k}^{2} \\
\ldots \\
\xi_{k}^{N}
\end{array}\right]}_{G_{k}}
$$

where $F$ is a constant known matrix whose entries $\bar{\rho}_{i}^{(j)}$ are the components of the vertices $\bar{\rho}_{i}$. At each discrete-time $k$, the matrix $E_{k}$ is known because $\rho_{k}$ is available. Hence, the matrix $G_{k}$ (and so the $\xi_{k}^{i}$ 's) are given by :

$$
G_{k}=F^{+} E_{k}
$$

where $F^{+}$is the pseudo-inverse of $F$ fulfilling the constraint $\xi_{k}^{i} \geq 0$.

\section{Determination of $\overline{A_{i}}$}

Substituting (4) into (3) yields :

$$
\mathcal{A}\left(\rho_{k}\right)=A_{0}+\sum_{j=1}^{L}\left(\sum_{i=1}^{N} \xi_{k}^{i} \bar{\rho}_{i}^{(j)}\right) A_{j}^{l c}
$$

Since $\sum_{i=1}^{N} \xi_{k}^{i}=1$ and $A_{0}$ is constant, $A_{0}=\sum_{i=1}^{N} \xi_{k}^{i} A_{0}$. Left factorizing the expression (7) by $\sum_{i=1}^{N} \xi_{k}^{i}$ yields :

$$
A\left(\rho_{k}\right)=\sum_{i=1}^{N} \xi_{k}^{i}(\underbrace{A_{0}+\sum_{j=1}^{L} \bar{\rho}_{i}^{(j)} A_{j}^{l c}}_{\overline{A_{i}}})
$$

Finally, identifying (8) with (2) gives the matrices $\overline{A_{i}}(i=$ $1, \ldots, N)$

$$
\overline{A_{i}}=A_{0}+\sum_{j=1}^{L} \bar{\rho}_{i}^{(j)} A_{j}^{l c}
$$

It is worth emphasizing that the description (1)-(2) includes some well-known special systems. Indeed, the case when $\mathcal{A}\left(y_{k}\right)=A$ is a constant dynamical matrix corresponds to a Lur'e system and the case when $\mathcal{A}\left(y_{k}\right)=A_{i}, E\left(y_{k}\right)=E_{i}$, some constant matrices, corresponds to a piecewise linear system. In next section, it is shown how such a result can be used to complete an adaptive synchronization of chaotic systems.

\section{ADAPTIVE SYNCHRONIZATION}

We propose an adaptive synchronization scheme with global convergence properties based upon a joint state and parameter estimation via a polytopic observer [8]. In the sequel, the system exhibiting a chaotic motion will be called the transmitter as usual.

\subsection{Observer design}

The transmitter under consideration is described by the following dynamics:

$$
\left\{\begin{array}{l}
x_{k+1}=A\left(y_{k}\right) x_{k}+f\left(y_{k}\right) \theta_{k}+g\left(y_{k}\right) \\
y_{k}=C x_{k}
\end{array}\right.
$$

with $x_{k} \in \Re^{n}, y_{k} \in \Re^{m}, f$ and $g$ two $n$-dimensional functions of $y_{k} . \theta_{k} \in \Re^{p}$ is the unknown parameter vector. Such a parameter has to be estimated and is assumed to be constant over a fixed interval of time. Furthermore, $A\left(y_{k}\right)$ and $f\left(y_{k}\right)$ are assumed to be bounded if $y_{k}$ is bounded.

By introducing an extended state vector denoted $\bar{x}_{k}$ composed by the states of the system and the parameter $\theta_{k}$, that is $\bar{x}_{k}=\left[x_{k}^{T} \theta_{k}^{T}\right]^{T}$, (10) can be rewritten :

$$
\left\{\begin{array}{l}
{\left[\begin{array}{l}
x_{k+1} \\
\theta_{k+1}
\end{array}\right]=\underbrace{\left[\begin{array}{cc}
A\left(y_{k}\right) & f\left(y_{k}\right) \\
\mathbf{0} & \mathbf{1}
\end{array}\right]}_{\mathcal{A}\left(y_{k}\right)}\left[\begin{array}{l}
x_{k} \\
\theta_{k}
\end{array}\right]+\left[\begin{array}{c}
g\left(y_{k}\right) \\
\mathbf{0}
\end{array}\right]} \\
y_{k}=\bar{C} \bar{x}_{k}
\end{array}\right.
$$

where $\bar{C}=\left[\begin{array}{ll}C & \mathbf{0}\end{array}\right], \mathbf{0}$ and $\mathbf{1}$ are respectively a null matrix and the identity matrix of compatible size. $\mathcal{A}$ is the dynamical matrix of the augmented system. When (10) exhibits a chaotic motion, all the components of both the state vector $x_{k}$ and the output $y_{k}$ are bounded. Furthermore, since $A\left(y_{k}\right)$ and $f\left(y_{k}\right)$ are assumed to be bounded if $y_{k}$ is bounded, it is always easy to check for a function $h$ such 
that $\mathcal{A}\left(y_{k}\right)=\mathcal{A}\left(\rho_{k}\right)$ with $\rho_{k}=h\left(y_{k}\right)$ and such that $\mathcal{A}$ is of class $C^{1}$ with respect to $\rho_{k}$. Thus, according to the result of Proposition 1, the matrix $\mathcal{A}\left(\rho_{k}\right)$ can be written in a polytopic way, that is:

$$
\mathcal{A}\left(\rho_{k}\right)=\sum_{i=1}^{N} \xi_{k}^{i}\left(\rho_{k}\right) \bar{A}_{i}
$$

where the $\xi_{i}\left(\rho_{k}\right)$ 's and the $\bar{A}_{i}$ 's are computed from (6) and (9).

Now, consider the observer with state vector $\hat{\bar{x}}_{k}=\left[\hat{x}_{k}^{T} \hat{\theta}_{k}^{T}\right]^{T}$ described by :

$$
\left\{\begin{array}{c}
{\left[\begin{array}{l}
\hat{x}_{k+1} \\
\hat{\theta}_{k+1}
\end{array}\right]=\mathcal{A}\left(\rho_{k}\right)\left[\begin{array}{l}
\hat{x}_{k} \\
\hat{\theta}_{k}
\end{array}\right]+} \\
L\left(\rho_{k}\right) \bar{C}\left[\begin{array}{l}
x_{k}-\hat{x}_{k} \\
\theta_{k}-\hat{\theta}_{k}
\end{array}\right]+\left[\begin{array}{c}
g\left(y_{k}\right) \\
\mathbf{0}
\end{array}\right] \\
\hat{y}_{k}=\bar{C} \hat{\bar{x}}_{k}
\end{array}\right.
$$

whose time-varying gain $L\left(\rho_{k}\right)$ is defined as follows :

$$
L\left(\rho_{k}\right)=\sum_{i=1}^{N} \xi_{k}^{i}\left(\rho_{k}\right) L_{i}
$$

Such an observer is called a polytopic observer since it involves matrices $\mathcal{A}$ and $L$ having a polytopic description. The resulting state reconstruction error $\epsilon_{k}=\bar{x}_{k}-\hat{\bar{x}}_{k}$, deduced from (11) and (13), is thus also described in a polytopic way

$$
\epsilon_{k+1}=\left(\sum_{i=1}^{N} \xi_{k}^{i}\left(\rho_{k}\right)\left(\bar{A}_{i}-L_{i} \bar{C}\right)\right) \epsilon_{k}
$$

We recall a recent general result ensuring the global stability of such a polytopic system :

Theorem 1 [8] Global stability of (15) is achieved whenever the feasibility condition of the following set of Linear Matrix Inequalities is satisfied:

$$
\left[\begin{array}{cc}
G_{i}+G_{i}^{T}-S_{i} & G_{i}^{T} \bar{A}_{i}-F_{i}^{T} \bar{C} \\
\bar{A}_{i}^{T} G_{i}-\bar{C}^{T} F_{i} & S_{j}
\end{array}\right]>0
$$

for all $(i, j) \in\{1, \ldots, N\} \times\{1, \ldots, N\}$

The $G_{i}$ 's, $S_{i}$ 's and $F_{i}$ 's are unknown matrices of appropriate dimensions. The resulting gains $L_{i}$ are given by $L_{i}=$ $G_{i}^{-T} F_{i}^{T}$.

Consequently, from Theorem 1, the $L_{i}$ 's ensure the global stability of (15) and so the global convergence toward zero of both errors $\left\|x_{k}-\hat{x}_{k}\right\|$ and $\left\|\theta_{k}-\hat{\theta}_{k}\right\|$. The proposed polytopic observer achieves a global adaptive synchronization.

\section{ILLUSTRATIVE EXAMPLE}

As mentioned in the introduction, adaptive synchronization can be used either to handle parameter mismatch problems or to retrieve the information in some communication scheme based on chaotic switching. The illustrative example deals with the later situation. The chaotic system acting as the transmitter corresponds to the Henon map :

$$
\left\{\begin{array}{l}
x_{k+1}^{(1)}=\theta_{k} \cdot\left(x_{k}^{(1)}\right)^{2}+x_{k}^{(2)}+1 \\
x_{k+1}^{(2)}=q \cdot x_{k}^{(1)} \\
y_{k}=x_{k}^{(1)}
\end{array} q=0.3\right.
$$

The scalar parameter $\theta_{k}(p=1)$ switches between $\theta_{\min }=$ -1.4 and $\theta_{\max }=-1.3$. Let note that the state $x_{k}^{(1)}$ is online available because $y_{k}=x_{k}^{(1)}$.

The extended state vector obeys the following equation:

$$
\left\{\begin{array}{l}
{\left[\begin{array}{l}
x_{k+1}^{(1)} \\
x_{k+1}^{(2)} \\
\theta_{k+1}
\end{array}\right]=\underbrace{\left[\begin{array}{lll}
0 & 1 & \left(x_{k}^{(1)}\right)^{2} \\
q & 0 & 0 \\
0 & 0 & 1
\end{array}\right]}_{\mathcal{A}\left(y_{k}\right)}\left[\begin{array}{l}
x_{k}^{(1)} \\
x_{k}^{(2)} \\
\theta_{k}
\end{array}\right]+\underbrace{\left[\begin{array}{l}
1 \\
0 \\
0
\end{array}\right]}_{g\left(y_{k}\right)}} \\
y_{k}=\underbrace{\left[\begin{array}{lll}
1 & 0 & 0
\end{array}\right]}_{\bar{C}}\left[\begin{array}{l}
x_{k}^{(1)} \\
x_{k}^{(2)} \\
\theta_{k}
\end{array}\right]
\end{array}\right.
$$

which is similar to (11) with

$$
A\left(y_{k}\right)=\left[\begin{array}{ll}
0 & 1 \\
q & 0
\end{array}\right] \text { and } f\left(y_{k}\right)=\left[\begin{array}{c}
\left(x_{k}^{(1)}\right)^{2} \\
0
\end{array}\right]
$$

Our goal is to reconstruct simultaneously the state $x_{k}^{(2)}$ and the parameter $\theta_{k}$. Taking $\rho_{k}=h\left(y_{k}\right)=\left(x_{k}^{(1)}\right)^{2}$ enforces $\mathcal{A}\left(y_{k}\right)$ to be expressed as a function of class $C^{1}$ with respect to $\rho_{k}$. Since $\rho_{k}$ is scalar $(L=1)$, one has $\rho_{k}^{(1)}=\rho_{k}$. Besides, (17) being chaotic, $x_{k}^{(1)}$ and so $\rho_{k}$ is bounded. Hence, assumptions of Proposition 1 are fulfilled which allows a polytopic description (2) for which the $\xi_{i}$ 's and the $\bar{A}_{i}$ 's have to be determined according to the constructive proof.

\section{Determination of $\xi_{i}$}

By simulating the chaotic system (17), it can be inferred that $\rho_{k}$ ranges between a minimum $\bar{\rho}_{1}^{(1)}=7.7 \cdot 10^{-6}$ and a maximum $\bar{\rho}_{1}^{(2)}=1.64$. Those extrema correspond to the vertices $(N=2)$ of the polytope wherein $\rho_{k}$ lies. Hence, $\rho_{k}$ can be expressed as in (4) or (5).

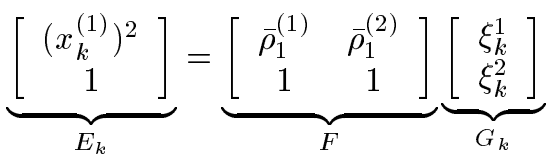


Note that for this particular case, the solutions of (19) leads to simple explicit forms :

$$
\xi_{k}^{1}=\frac{\rho_{k}-\bar{\rho}_{1}^{(2)}}{\bar{\rho}_{1}^{(1)}-\bar{\rho}_{1}^{(2)}}
$$

and $\xi_{k}^{2}=1-\xi_{k}^{1}$ since $\sum_{i=1}^{N} \xi_{k}^{i}=1$.

Determination of $\bar{A}_{i}$

The $\bar{A}_{i}$ 's are computed in a straightforward way according to (9) and yields :

$$
\bar{A}_{1}=\left[\begin{array}{ccc}
0 & 1 & 7.7 \cdot 10^{-6} \\
0.3 & 0 & 0 \\
0 & 0 & 1
\end{array}\right] \quad \bar{A}_{2}=\left[\begin{array}{ccc}
0 & 1 & 1.64 \\
0.3 & 0 & 0 \\
0 & 0 & 1
\end{array}\right]
$$

Determination of $L_{i}$

The gain $L\left(\rho_{k}\right)$ verifies (14). The $\xi_{k}^{i}$ 's are the same than the ones involved in the polytopic decomposition (12) and resulting from the solution of (19). The $L_{i}$ 's are derived from the solution of the LMI (16) with $\bar{A}_{i}$ given in (20). The resolution can be performed thanks to standard LMI solvers.

$$
L_{1}=\left[\begin{array}{c}
-0.0089 \\
0.3000 \\
0.2741
\end{array}\right] \text { and } L_{2}=\left[\begin{array}{l}
0.5677 \\
0.3000 \\
0.3387
\end{array}\right]
$$

The design of the polytopic observer (13) is now completed. The figure 1 shows the convergence of the estimated parameter $\hat{\theta}_{k}$ toward the true parameter $\theta_{k}$ which switches periodically between two distinct values and the convergence of the error of synchronization toward zero. The simulations are consistent with the theoretical results. Let recall that since the convergence is global, it is achieved whatever be the initial conditions.

\section{CONCLUSION}

A scheme ensuring a global adaptive synchronization by a joint state and parameter estimation has been proposed as an alternative to the standard Extended Kalman Filter. The advantage lies in the fact that the design does no longer require a first order approximation, preventing some possible bad convergence properties. The gain of the polytopic observer is easily computed from the resolution of a Linear Matrix Inequalities set. Besides, the novelty of such an approach stems from the fact that the design takes into account some chaos specificities as the bounds of the state vector in the state space.
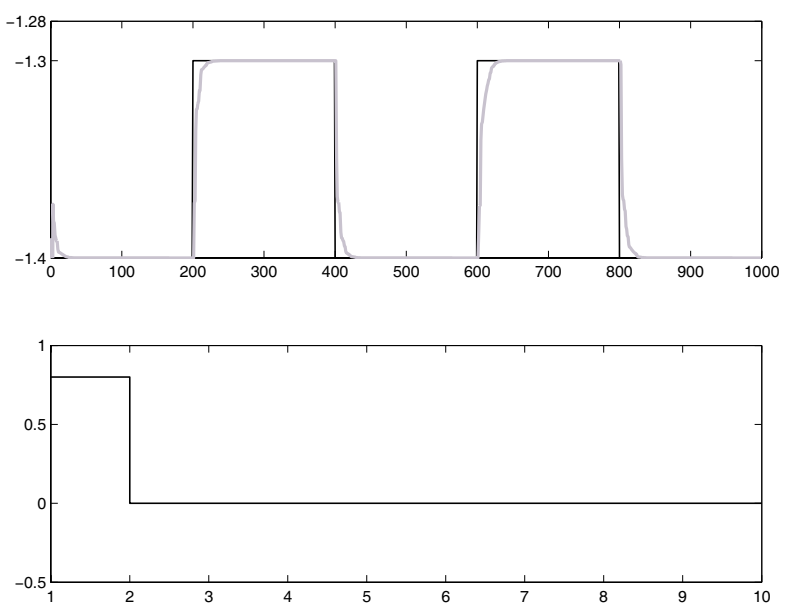

Fig. 1. Black $\theta_{k}$, gray $\hat{\theta}_{k}$ (up). Error of synchronization $\epsilon_{k}$ (down)

\section{REFERENCES}

[1] C. W. Wu, T. Yang, and L. O Chua, "On adaptive synchronization and control of nonlinear dynamical systems," International Journal of Bifurcation and Chaos, vol. 6, no. 3, pp. 455-471, 1996.

[2] A. L. Fradkov and A. Y. Markov, "Adaptive synchronization of chaotic systems based on speed-gradient method and passification," IEEE Trans. Circuits. Syst. I: Fundamental Theo. Appl, vol. 44, no. 10, pp. 905-912, Oct. 1997.

[3] H. Dedieu and M. Ogorzalek, "Identifiability and identification of chaotic systems based on adaptative synchronization," IEEE Trans. Circuits. Syst. I: Fundamental Theo. Appl, vol. 44, no. 10, pp. 948-962, October 1997.

[4] K-Y Lian, P. Liu, T-S Chiang, and C-S Chiu, "Adaptive synchronization design for chaotic systems via a scalar driving signal," IEEE Trans. Circuits. Syst. I: Fundamental Theo. Appl, vol. 49, no. 1, pp. 17-25, January 2002.

[5] R. Marino and P. Tomei, "Adaptive observers with arbitrary exponential rate of convergence for nonlinear systems," IEEE Trans. on Automatic Control, vol. 40, pp. 1300-1304, July 1995.

[6] D. J. Sobiski and J. S. Thorp, "Pdma-1: chaotic communication via the extended kalman filter," IEEE Trans. On Circuits and Systems - I Fund. Theory and Appl., vol. 45, no. 2, pp. 194-197, 1998.

[7] C. Cruz and H. Nijmeijer, "Synchronization through filtering," International Journal of Bifurcation and Chaos, vol. 110, no. 4, pp. 763-775, 2000.

[8] G. Millerioux and J. Daafouz, "Polytopic observer for global synchronization of systems with output measurable nonlinearities," International Journal of Bifurcation and Chaos, vol. 13, no. 3, pp. 703-712, March 2003. 